\title{
Preliminary determinations of the masses of the neutron star and mass donor in the high mass $X$-ray binary system EXO 1722-363* (Research Note)
}

\author{
A. B. Mason ${ }^{1}$, A. J. Norton ${ }^{1}$, J. S. Clark ${ }^{1}$, I. Negueruela ${ }^{2}$, and P. Roche ${ }^{1,3,4}$ \\ 1 Department of Physics \& Astronomy, The Open University, Milton Keynes MK7 6AA, UK \\ e-mail: a.mason@open.ac.uk \\ 2 Departamento de Física, Ingeniería de Sistemas y Teoría de la Señal, Universidad de Alicante, Apdo. 99, E03080 Alicante, Spain \\ 3 School of Physics \& Astronomy, Cardiff University, The Parade, Cardiff, CF24 3AA, UK \\ 4 Division of Earth, Space \& Environment, University of Glamorgan, Pontypridd, CF37 1DL, UK
}

Received 1 October 2009 / Accepted 17 November 2009

\section{ABSTRACT}

\begin{abstract}
Aims. We intended to measure the radial velocity curve of the supergiant companion to the eclipsing high mass X-ray binary pulsar EXO 1722-363 and hence determine the stellar masses of the components.

Methods. We used a set of archival $K_{\mathrm{s}}$-band infrared spectra of the counterpart to EXO 1722-363 obtained using ISAAC on the VLT, and cross-correlated them in order to measure the radial velocity of the star.

Results. The resulting radial velocity curve has a semi-amplitude of $24.5 \pm 5.0 \mathrm{~km} \mathrm{~s}^{-1}$. When combined with other measured parameters of the system, this yields masses in the range $1.5 \pm 0.4-1.6 \pm 0.4 M_{\odot}$ for the neutron star and $13.6 \pm 1.6-15.2 \pm 1.9 M_{\odot}$ for the B0-1 Ia supergiant companion. These lower and upper limits were obtained under the assumption that the system is viewed edge-on $\left(i=90^{\circ}\right)$ for the lower limit and the supergiant fills its Roche lobe $(\beta=1)$ for the upper limit respectively. The system inclination is constrained to $i>75^{\circ}$ and the Roche lobe-filling factor of the supergiant is $\beta>0.9$. Additionally we were able to further constrain our distance determination to be $7.1 \leq d \leq 7.9 \mathrm{kpc}$ for EXO 1722-363. The X-ray luminosity for this distance range is $4.7 \times 10^{35} \leq L_{\mathrm{X}} \leq 9.2 \times 10^{36} \mathrm{erg} \mathrm{s}^{-1}$.

Conclusions. EXO 1722-363 therefore becomes the seventh of the ten known eclipsing X-ray binary pulsars for which a dynamical neutron star mass solution has been determined. Additionally EXO 1722-363 is the first such system to have a neutron star mass measurement made utilising near-infrared spectroscopy.
\end{abstract}

Key words. pulsars: general - binaries: eclipsing - binaries: general - X-rays: binaries - stars: individual: EXO 1722-363 stars: neutron

\section{Introduction}

The precise form of the neutron star (NS) equation of state is still unknown. Despite much theoretical work aimed at determining this fundamental aspect of astrophysics, to eliminate some of the contending theories we must turn to observational data. Presently the only means of determining the mass of neutron stars in accretion driven systems is by observing eclipsing $\mathrm{X}$-ray binary pulsars. Unfortunately only 10 such systems are currently known, and only 6 of these have previously had mass measurements made (e.g. Ash et al. 1999; Quaintrell et al. 2003; Val Baker et al. 2005). In this paper we present the preliminary results from our on-going work on the mass of the High Mass X-ray Binary (HMXB) accretion driven pulsar EXO 1772-363. The counterpart star within this HMXB is heavily obscured and reddened, necessitating for the first time the utilisation of near-infrared spectroscopy to construct the Radial Velocity (RV) curve in order to obtain an accurate mass solution.

Observations made of EXO 1722-363 (alternatively designated IGR J17252-3616), in 1987 by the Ginga X-ray satellite were the first to detect pulsations. These pulsations were

* Based on observations carried out at the European Southern Observatory under programme ID 077.B-0872(A). found to have a $413.9 \pm 0.2 \mathrm{~s}$ period (Tawara et al. 1989). Over an $8 \mathrm{~h}$ period the source appeared to vary substantially in flux, decreasing from 2 mcrab to $0.2-0.3$ mcrab within the 6-21 keV band. It was subsequently found that the X-ray flux remained persistent from $20-60 \mathrm{keV}$, however a cutoff point was found above this flux level in which the source was undetectable. EXO 1722-363 at maximum flux and assuming a distance of $10 \mathrm{kpc}$, had a luminosity calculated as $5 \times 10^{36} \mathrm{erg} \mathrm{s}^{-1}$ (Tawara et al. 1989). Later observations by the Rossi X-ray Timing Explorer (RXTE) revealed the eclipsing nature of this system with the eclipse duration determined as $1.7 \pm 0.1$ days (Corbet et al. 2005). Subsequent observations by INTEGRAL followed up by XMM-Newton in 2004 led to a further refinement of the spin and orbital periods to $413.851 \pm 0.004 \mathrm{~s}$ and $9.7403 \pm 0.0004$ days respectively (Thompson et al. 2007).

XMM-Newton observations allowed the source position to be determined more precisely (with an uncertainty of $4^{\prime \prime}$ ) at $\mathrm{RA}(2000.0)=17^{\mathrm{h}} 25^{\mathrm{m}} 11.4^{\mathrm{s}}$ and $\mathrm{Dec}=-36^{\circ} 16^{\prime} 58.6^{\prime \prime}$. EXO 1722-363 lies within the Galactic plane and as it is heavily reddened, unsurprisingly the counterpart star could not be detected optically. An infrared counterpart was found lying $1^{\prime \prime}$ from the X-ray source position (Zurita Heras et al. 2006) with a corresponding entry in the 2 MASS catalogue, 
Table 1. The phase, radial velocity and telluric standard for each EXO 1722-363 archive spectrum.

\begin{tabular}{cccccc}
\hline \hline Mid-point of Observations (UT) & HJD & Phase & Radial velocity / km s & Telluric Std & Telluric Spec. Type \\
\hline 2006 May 24.340 & 2453879.83 & 0.130 & $-18.61 \pm 11.8$ & Hip 093225 & B4V \\
2006 Jun. 22.025 & 2453908.53 & 0.077 & $-11.74 \pm 11.8$ & Hip 088109 & B5II \\
2006 Jun. 29.233 & 2453915.74 & 0.817 & $-21.39 \pm 11.8$ & Hip 070148 & B8III \\
2006 Jul. 14.167 & 2453930.67 & 0.350 & $14.92 \pm 11.8$ & Hip 089960 & B6V \\
2006 Jul. 17.248 & 2453933.75 & 0.666 & $-29.22 \pm 11.8$ & Hip 087616 & B9IV/V \\
2006 Jul. 20.122 & 2453936.63 & 0.961 & $-14.95 \pm 11.8$ & Hip 090336 & B7III \\
2006 Jul. 22.112 & 2453938.62 & 0.165 & $-10.09 \pm 11.8$ & Hip 094859 & B5V \\
2006 Jul. 24.115 & 2453940.62 & 0.371 & $21.21 \pm 11.8$ & Hip 090336 & B7III \\
2006 Jul. 25.105 & 2453941.61 & 0.473 & $6.89 \pm 11.8$ & Hip 089960 & B6V \\
2006 Aug. 01.111 & 2453948.57 & 0.188 & $-3.83 \pm 11.8$ & Hip 085548 & B9II \\
2006 Aug. 02.113 & 2453949.62 & 0.295 & $28.06 \pm 11.8$ & Hip 085548 & B9II \\
2008 May 17.115 & $\mathbf{2 4 5} \mathbf{4 6 0 3 . 6 2}$ & $\mathbf{0 . 4 3 9}$ & $\mathbf{3 0 . 9 5} \pm \mathbf{1 1 . 8}$ & Hip 085008 & B5V \\
\hline
\end{tabular}

2MASS J17251139-3616575 (with $J H K$ magnitudes $J=14.2$, $H=11.8$ and $\left.K_{\mathrm{s}}=10.7\right)$. Examination of near infrared $K$-band spectra obtained with the ESO ISAAC instrument led to our determination of the spectral classification of the mass donor as B0-B1 Ia (Mason et al. 2009).

\section{Observations and data reduction}

In our previous work, we only had single epoch $K_{\mathrm{s}}$-band spectra of the mass donor in EXO 1722-363, but in order to determine a dynamical mass solution, radial velocities at a range of orbital phases are required. Fortunately we were able to locate a series of $K_{\mathrm{s}}$-band spectra held within the ESO Archive ${ }^{1}$ which were obtained over 26 nights between 24th May, 2006 and 4th August, 2006. 26 pairs of spectra were centred on $2.1 \mu \mathrm{m}$ and 26 pairs centred on $2.2 \mu \mathrm{m}$. No observations of radial velocity standards appear to have been taken with any of the science spectra, however there are telluric standards available to enable removal of atmospheric features from the target spectra.

The data were reduced using the ISAAC pipeline ${ }^{2}$ in conjunction with the data browsing tool GASGANO ${ }^{3}$. Unfortunately within the $2.2 \mu \mathrm{m}$ EXO 1722-363 dataset, the counterpart to EXO 1722-363 had been incorrectly identified and the telescope had been mis-pointed, so the spectra were unusable. Additionally only 11 of the 26 spectra from the dataset centred on $2.1 \mu \mathrm{m}$ turned out to be of sufficient quality to derive a radial velocity measurement (See Table 1 ). The usable spectra were made in the SW MRes mode with a $0.6^{\prime \prime}$ slit. Resulting in spectra with a high $\mathrm{S} / \mathrm{N}$ and resolution $(R \approx 4200)$. The integration time for each pair of spectra was $700 \mathrm{~s}$, with the resulting data having a count rate below 10000 ADU; therefore no correction for non-linearity was necessary.

Flatfields were reduced using the pipeline recipe isaac_spc_flat and combined to produce a master flatfield. The wavelength calibration and ISAAC slit curvature distortion was computed using $\mathrm{OH}$ skylines using the pipeline recipe isaac_spc_arc. Spectra produced by ISAAC have a high degree of curvature, to remove this the pipeline recipe isaac_spc_startrace computes the spectra curvature using both images and spectra of a star moving across the slit. Science spectra were obtained using the nodding technique; unfortunately only one nod was performed during the original

\footnotetext{
${ }^{1}$ http://archive.eso.org/eso/eso_archive_main.html

2 http://www.eso.org/sci/data-processing/software/ pipelines/isaac/isaac-pipe-recipes.html

3 http://www.eso.org/sci/data-processing/software/ gasgano/
}

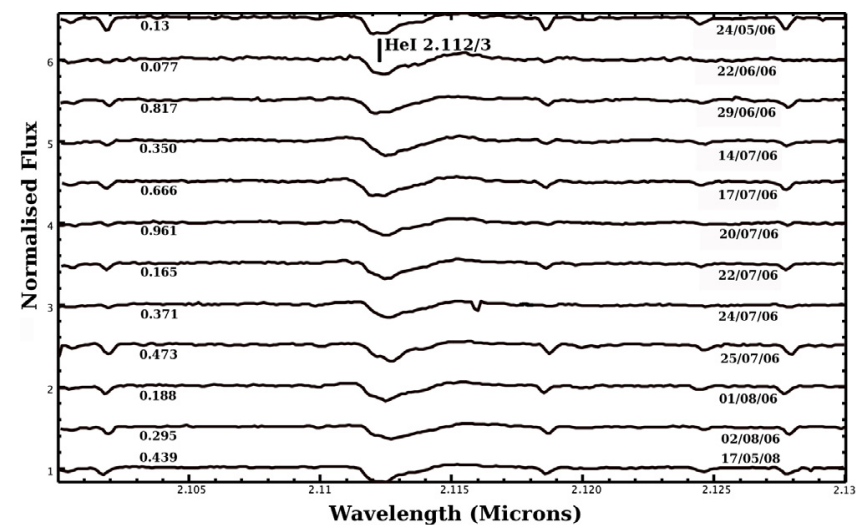

Fig. 1. Continuum normalised $K_{\mathrm{s}}$-band spectra centred on $2.1 \mu \mathrm{m}$ of EXO 1722-363 in order of date from top to bottom.

observation, which we believe to be less than optimal. The two nodded science frames were then reduced using the products of the pipeline calibration recipes to produce a final reduced science spectrum. This process was then repeated for each telluric standard. Telluric correction was then made using the standards shown in Table 1. All spectra were reduced using standard IRAF $^{4}$ routines; Fig. 1 shows the stacked continuum normalised spectra ordered by date of observation.

\section{Data analysis}

Radial velocities were determined by cross-correlating the region around the HeI 2.112/3 $\mu \mathrm{m}$ absorption line in each of the 11 archive spectra against the high signal-to-noise $K_{\mathrm{s}}$-band spectrum of EXO 1722-363, which we had previously obtained for spectral classification purposes (Mason et al. 2009). The resulting velocities were then corrected to the solar system barycentre and are reported in Table 1. The spectrum highlighted in bold is that used as the reference spectrum (our previously obtained high signal-to-noise $K_{\mathrm{s}}$-band spectrum of EXO 1722-363 Mason et al. 2009). In obtaining these final velocities, we determined the absolute velocity of the reference spectrum by fitting the positions of its absorption lines.

From X-ray data there is no evidence that EXO 1722-363 has anything other than a circular orbit (Thompson et al. 2007),

\footnotetext{
${ }^{4}$ IRAF is distributed by the National Optical Astronomy Observatory, which is operated by the Association of Universities for Research in Astronomy, Inc., under cooperative agreement with the National Science Foundation.
} 


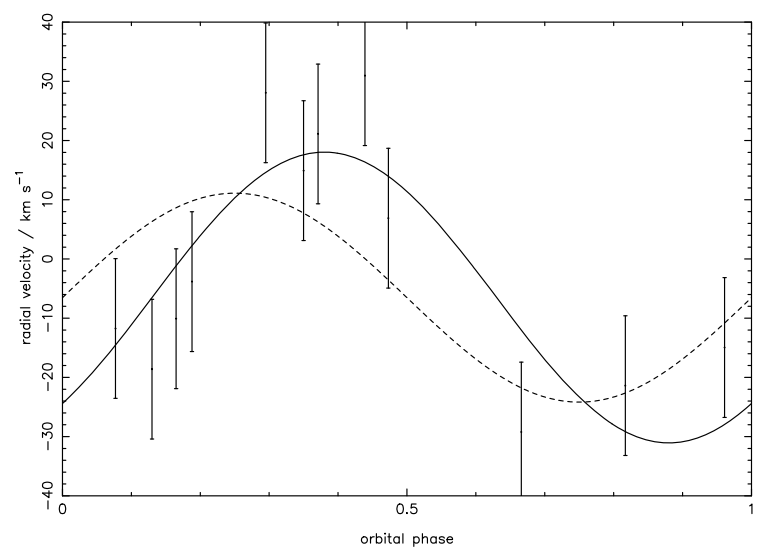

Fig. 2. Radial velocity data for the supergiant star in EXO 1722-363. The solid line is the best fitting sinusoid with three free parameters, the dashed line is that with a fixed zero phase in line with the published ephemeris. The orbital phase is based upon the ephemeris of Thompson et al. (2007).

so we fitted the radial velocities of the supergiant star with a simple sinusoidal solution. The ephemeris of Thompson et al. (2007) specifies the epoch of mid-eclipse as

$$
T(\text { HJD })=53761.68(4)+9.7403(4) N
$$

where $N$ is the cycle number and uncertainties in brackets refer to the last decimal place quoted. At the epoch of our observations, the accumulated uncertainty in phase is formally only $\sim 0.005$, but nonetheless we fitted our data with two models, in one of which the zero phase was a free parameter and in the other of which it was not.

Fitting our data with a sinusoid with just two free parameters (RV amplitude and systemic velocity) yielded an amplitude of $17.6 \pm 7.7 \mathrm{~km} \mathrm{~s}^{-1}$ and a systemic velocity of $-6.5 \pm 5.6 \mathrm{~km} \mathrm{~s}^{-1}$. In order to achieve a reduced chi-squared of unity, the uncertainties on each RV data point had to be scaled to $\pm 17.5 \mathrm{~km} \mathrm{~s}^{-1}$. In comparison, fitting our data with a sinusoid with three free parameters (i.e. with the addition of zero phase as a free parameter) gave an amplitude of $24.6 \pm 5.0 \mathrm{~km} \mathrm{~s}^{-1}$, a systemic velocity of $-6.5 \pm 3.8 \mathrm{~km} \mathrm{~s}^{-1}$ and a phase shift of $-0.13 \pm 0.03$. In this case, a reduced chi-squared of unity was achieved by scaling the uncertainties on each point to $\pm 11.8 \mathrm{~km} \mathrm{~s}^{-1}$. Although the bestfit phase offset is discrepant with the accumulated phase uncertainty of the ephemeris, we prefer this fit and use the data from it subsequently; both fits are shown in Fig. 2 in which the value for $K_{\mathrm{O}}$ is that resulting from fitting the radial velocities including a phase shift.

The masses of the system components may be determined as follows. The mass ratio of the system $q$ is equal to the ratio of the semi-amplitudes of the radial velocities for each star

$q=\frac{M_{\mathrm{X}}}{M_{\mathrm{O}}}=\frac{K_{\mathrm{O}}}{K_{\mathrm{X}}}$

where $M_{\mathrm{X}}$ and $M_{\mathrm{O}}$ are the masses of the neutron star and supergiant star respectively, and $K_{\mathrm{X}}$ and $K_{\mathrm{O}}$ are the corresponding semi-amplitudes of their radial velocities. In addition, for circular orbits,

$M_{\mathrm{O}}=\frac{K_{\mathrm{X}}^{3} P}{2 \pi G \sin ^{3} i}(1+q)^{2}$

and similarly

$M_{\mathrm{X}}=\frac{K_{\mathrm{O}}^{3} P}{2 \pi G \sin ^{3} i}\left(1+\frac{1}{q}\right)^{2}$ where $i$ is the inclination to the plane of the sky and $P$ is the orbital period. For EXO 1722-363, X-ray pulse timing delays yield the value of $K_{\mathrm{X}}$ as $226.1 \pm 6.7 \mathrm{~km} \mathrm{~s}^{-1}$ (Thompson et al. 2007). A value for the system inclination can be found from the geometric relation

$\sin i \approx \frac{\left[1-\beta^{2}\left(\frac{R_{\mathrm{L}}}{a}\right)^{2}\right]^{1 / 2}}{\cos \theta_{\mathrm{e}}}$

where $\theta_{\mathrm{e}}$ is the eclipse half-angle, $R_{\mathrm{L}}$ is the Roche lobe radius of the supergiant, $\beta$ is the ratio the supergiant's radius to that of its Roche lobe and $a$ is the separation between the centres of mass of the two stars. The Roche lobe radius may be approximated by

$$
\frac{R_{\mathrm{L}}}{a} \approx A+B \log q+C \log ^{2} q
$$

where the constants have been determined by Joss \& Rappaport (1984) as

$$
\begin{aligned}
& A \approx 0.398-0.026 \Omega^{2}+0.004 \Omega^{3} \\
& B \approx-0.264+0.052 \Omega^{2}-0.015 \Omega^{3} \\
& C \approx-0.023-0.005 \Omega^{2}
\end{aligned}
$$

$\Omega$ is the ratio of the spin period of the supergiant to its orbital period. For EXO 1722-363 we have assumed that the supergiant is close to Roche lobe-filling and is rotating synchronously with the orbit, so $\Omega=1$ (although we note this assumption may not be entirely correct in this case), and the eclipse half angle is measured using RXTE data to be $\theta_{\mathrm{e}}=31.8^{\circ} \pm 1.8^{\circ}$ (Corbet et al. 2005).

Hence the above set of equations allow the masses of the two stars to be determined in two limits. First, assuming that the supergiant fills its Roche lobe (in which case $\beta=1$ ) we can find a lower limit to the system inclination $i$ and upper limits to the stellar masses. Secondly, assuming that the system is viewed edge-on (in which case $i=90^{\circ}$ ) we can find a lower limit to the Roche lobe filling factor $\beta$ and lower limits to the stellar masses. Unfortunately, the spectra we have obtained from the ESO archive are not of sufficient quality to conduct a non-LTE model atmosphere analysis and hence make an accurate determination of the stellar radius which would break this degeneracy.

In order to propagate the uncertainties in each parameter, we performed a Monte Carlo analysis of the above equations to determine the system masses. The results in each limit are shown in Table 2, and of course masses lying between the extremes are also valid and correspond to values of $i$ and $\beta$ between their extremes.

\section{Discussion}

Although the 11 spectra reported here are of relatively low quality, and few in number, they still allow us to make a preliminary determination of the orbit of the supergiant in EXO 1722-363 and make a first measurement of the dynamical masses of the stellar components. The results are encouraging for a number of reasons. First, the resulting neutron star mass is consistent with the canonical mass of $1.4 M_{\odot}$ measured in most other eclipsing HMXBs, except for that in Vela X-1, (Quaintrell et al. 2003). Second, the measured mass and radius of the supergiant, $M \sim 13-15 M_{\odot}$ and $R \sim 25-28 R_{\odot}$, support the B01 Ia spectral classification that we have previously determined 
Table 2. System parameters for EXO 1722-363.

\begin{tabular}{|c|c|c|c|}
\hline Parameter & \multicolumn{2}{|c|}{ Value } & Ref. \\
\hline Observed & & \\
\hline$a_{\mathrm{X}} \sin i /$ lt $\mathrm{s}$ & & & [1] \\
\hline$P / \mathrm{d}$ & \multicolumn{2}{|c|}{$\begin{array}{c}101 \pm 1 \\
9.7403 \pm 0.0004\end{array}$} & [1] \\
\hline$T_{90} / \mathrm{HJD}$ & \multicolumn{2}{|c|}{$53761.68 \pm 0.04$} & [1] \\
\hline$e$ & \multicolumn{2}{|c|}{$<0.19$} & [1] \\
\hline$\theta_{\mathrm{e}} / \operatorname{deg}$ & \multicolumn{2}{|c|}{$31.8 \pm 1.8$} & [2] \\
\hline$K_{\mathrm{O}} / \mathrm{km} \mathrm{s}^{-1}$ & \multicolumn{2}{|c|}{$24.5 \pm 5.0$} & [3] \\
\hline Assumed & \multirow{3}{*}{\multicolumn{2}{|c|}{$=1$}} & \\
\hline$\Omega$ & & & \\
\hline Inferred & & & \\
\hline$K_{\mathrm{X}} / \mathrm{km} \mathrm{s}^{-1}$ & \multicolumn{2}{|c|}{$226.1 \pm 6.7$} & \\
\hline$q$ & \multicolumn{2}{|c|}{$0.107 \pm 0.022$} & \\
\hline$\beta$ & 1.000 & $0.916 \pm 0.047$ & \\
\hline$i / \operatorname{deg}$ & $75.2 \pm 4.6$ & 90.0 & \\
\hline$M_{\mathrm{X}} / M_{\odot}$ & $1.63 \pm 0.38$ & $1.46 \pm 0.38$ & \\
\hline$M_{\mathrm{O}} / M_{\odot}$ & $15.2 \pm 1.9$ & $13.6 \pm 1.6$ & \\
\hline$a / R_{\odot}$ & $49.1 \pm 9.1$ & $47.3 \pm 8.8$ & \\
\hline$R_{\mathrm{L}} / R_{\odot}$ & $28.0 \pm 5.3$ & $27.0 \pm 5.0$ & \\
\hline$R_{\mathrm{O}} / R_{\odot}$ & $28.0 \pm 5.3$ & $24.7 \pm 4.7$ & \\
\hline
\end{tabular}

Notes. [1] Thompson et al. 2007; [2] Corbet et al. 2005; [3] this paper.

(Mason et al. 2009). This is illustrated by the HertzsprungRussell diagram plotted in Fig. 3, which shows a close correspondence between the system primary and the properties of other galactic field BSGs (Searle et al. 2008). While the similarity in temperature is to be expected - the value for the primary was adopted on the basis of its spectral type, which in turn has been calibrated by the analysis of Searle et al. (2008), the radii for the field BSGs were determined via non-LTE model atmosphere analysis, while that for the primary is instead determined dynamically.

The measurement of the stellar radius and hence bolometric luminosity, has in turn allowed a more precise determination of the distance to the system by comparison to its observed photometric magnitude and reddening. The refined distance to EXO $1722-363$ of $7.1-7.9 \mathrm{kpc}$ results in an X-ray luminosity ranging from $L_{X_{\min }}=0.47 \times 10^{36}$ to $L_{X_{\max }}=9.2 \times 10^{36} \mathrm{erg} \mathrm{s}^{-1}$. However, due to the nature of the archive observations used for this work, the uncertainties on the mass and radius parameters are still rather large; it is our intention in the near future to propose and obtain more accurate VLT/ISAAC observations to further constrain the orbital solution parameters for this HMXB system.

Finally, comparison to evolutionary tracks in Fig. 3 might suggest the primary had an initial progenitor mass of $\sim 35-40 M_{\odot}$ and hence the neutron star originated in a more massive star. However we caution that the binary is highly likely to have undergone at least one episode of mass transfer in the past, rendering such a conclusion highly uncertain. As an exemplar we cite GX 301-2, a HMXB composed of a NS and a B hypergiant with a spectroscopic mass of $43 \pm 10 M_{\odot}$ (Kaper et al. 2006). However (Wellstein \& Langer 1999) propose a formation scenario in which two stars of comparable initial masses evolved via quasi conservative mass transfer into the current configuration post supernova; hence determining progenitor masses for both primary and neutron star based on the current system parameters is non trivial.

Acknowledgements. A.B.M. acknowledges support from an STFC studentship. J.S.C. acknowledges support from an RCUK fellowship. This research is

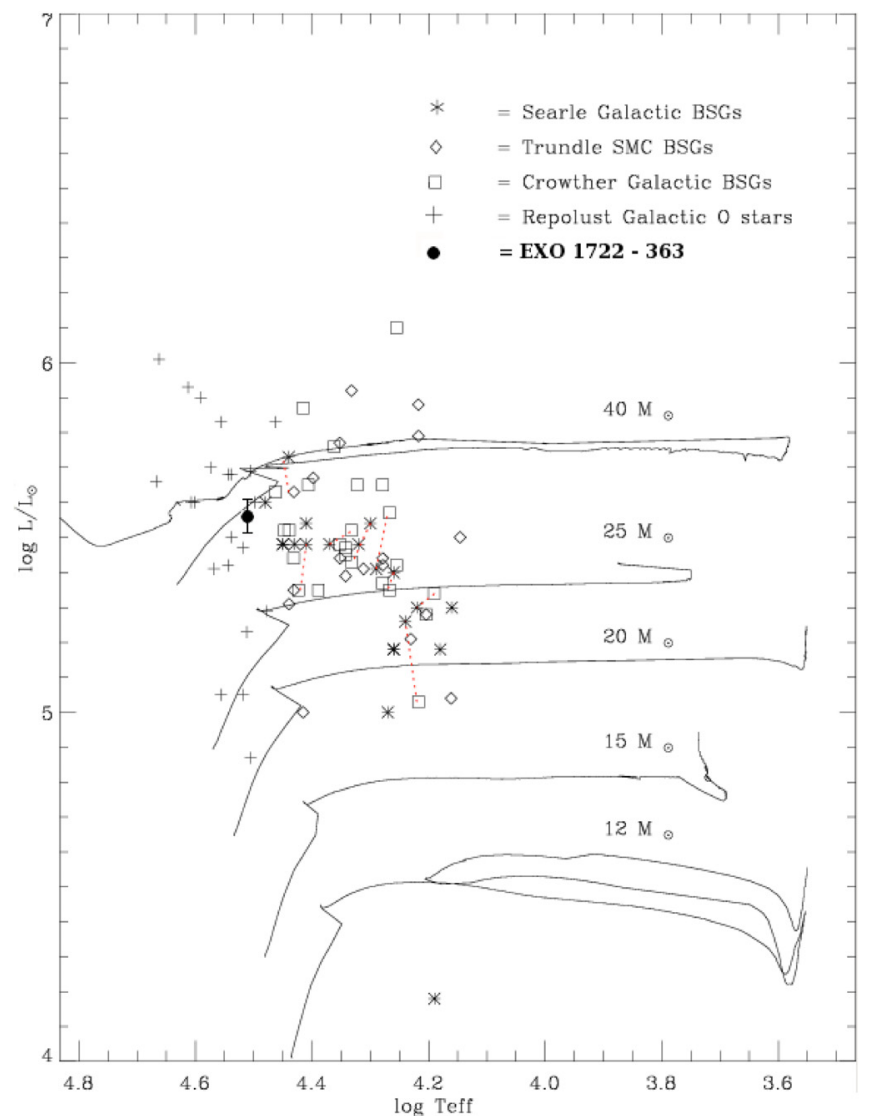

Fig. 3. Position of EXO 1722-363 on the Hertzsprung-Russell (Searle et al. 2008) diagram alongside a sample of $\mathrm{O}$ and $\mathrm{B}$ supergiants from differing locations, Galactic B supergiants, Crowther et al. (2006), SMC B supergiants (Trundle et al. 2004; Trundle \& Lennon 2005) and Galactic O stars, Repolust et al. (2004). These are overplotted together with solar metallicity evolutionary tracks from Meynet \& Maeder (2000). Also shown is the lower and upper limits on the luminosity of EXO 1772-363.

partially supported by grants AYA2008-06166-C03-03 and Consolider-GTC CSD-2006-00070 from the Spanish Ministerio de Ciencia e Innovación (MICINN). Based on observations carried out at the European Southern Observatory, Chile through programme ID 077.B-0872(A).

\section{References}

Ash, T. D. C., Reynolds, A. P., Roche, P., Norton, A. J., Still, M. D., \& Morales-Rueda, L. 1999, MNRAS, 307, 357

Corbet, R. H. D., Markwardt, C. B., \& Swank, J. H. 2005, ApJ, 633, 377

Crowther, P. A., Lennon, D. J., \& Walborn, N. R. 2006, A\&A, 446, 279

Joss, P. C., \& Rappaport, S. A. 1984, ARA\&A, 22, 537

Kaper, L., van der Meer, A., \& Najarro, F. 2006, A\&A, 457, 595

Mason, A. B., Clark, J. S., Norton, A. J., Negueruela, I., \& Roche, P. 2009, A\&A, 505,281

Meynet, G., \& Maeder, A. 2000, A\&A, 361, 101

Quaintrell, H., Norton, A. J., Ash, T. D. C., et al. 2003, A\&A, 401, 313

Repolust, T., Puls, J., \& Herrero, A. 2004, A\&A, 415, 349

Searle, S. C., Prinja, R. K., Massa, D., \& Ryans, R. 2008, A\&A, 481, 777

Tawara, Y., Yamauchi, S., Awaki, H., et al. 1989, PASJ, 41, 473

Thompson, T. W. J., Tomsick, J. A., in 't Zand, J. J. M., Rothschild, R. E., \&

Walter, R. 2007, ApJ, 661, 447

Trundle, C., \& Lennon, D. J. 2005, A\&A, 434, 677

Trundle, C., Lennon, D. J., Puls, J., \& Dufton, P. L. 2004, A\&A, 417, 217

Val Baker, A. K. F., Norton, A. J., \& Quaintrell, H. 2005, A\&A, 441, 685

Wellstein, S., \& Langer, N. 1999, A\&A, 350, 148

Zurita Heras, J. A., de Cesare, G., Walter, R., et al. 2006, A\&A, 448, 261 